Send your letters to the Editor,

British Dental Journal,

64 Wimpole Street

London

W1G 8YS

E-mailbdj@bda.org

Priority will be given to letters less than 500 words long.

Authors must sign the letter, which

may be edited for reasons of space.

\section{POSTURE TECHNIQUE}

Sir, I compliment A. Gandavani et al. (BDJ 2007; 203: 601-605) for highlighting the importance of correct posture in preventing back pain. I am concerned about some aspects of the study.

For example Figures 3 and 4 are not truly comparable. The student in Figure 3 on the BSS has the 'patient's head' at the correct working height (as shown by her upward-sloping forearms). However, the one on the CS in Figure 4 has the patient's head far too low; his stool is not high enough (so producing the 90 degree flexion of knees and hips rightly condemned in the article); his stool seat slopes down backwards and the stool back is tilted so far back as to make it useless. Little wonder he is in such distorted posture.

Any one of these errors would produce the kyphosis and slumping referred to in the study. Combined in one dentist they are a disaster.

On the other hand I can guarantee that if this student's stool was raised to a correct height and its seat tilted slightly forward, this would produce the correct knee and femur/trunk angles of more than 90 degrees. The dental chair should then be raised to place the patient's mouth at the correct height at the student's mid-sternal level. Finally if the stool back rest were brought forward into firm contact with his lower back to reciprocate his lumbar lordosis, his posture would be as good as the student on the BSS. No equipment will work if not used correctly!

Excellent though this study is one must also look at stools in a general practice context.

In my experience there are some disadvantages of the BSS:
1. Sitting for long periods with the thighs widely splayed seems to put pressure on the hip joints. I have met two dentists recently who have had to cease using a BSS due to hip pain

2. This thigh splay prevents the nurse from sitting close enough to the patient forcing her to lean over both forwards and sideways (nurses also have backs)

3. Some dental chairs do not go high enough to put the mouth at a dentist's focal distance causing him or her to overflex

4. Nurses cannot sit high enough when the dentist sits at the height required by a BSS. Many have to stand.

In conclusion - even the most perfect stool will not be totally effective if all the other techniques needed to work in perfect posture are not applied.

E. Paul By email

Dr Amar Gandavani responds: We wish to thank Dr Paul for his interest and for his positive and helpful comments. In response to his comment about figures: the students were initially trained in properly adjusting and using their respective seats (Bambach Seat [BS] and Conventional Seat [CS]) with the correct operating posture before the commencement of the study. The students using the $B S$ developed healthy posture, whereas the students using the CS developed a less ideal posture.

Regarding CS producing 90 degree flexion of the knees: Mandal (1984) has clearly identified that using a CS keeps the knees in 90 degree flexion, the pelvis is tilted posteriorly, and the body is tilted away from the work surface, which is compensated by increasing the flexion of the lumbar spine (slumped sitting). This posture was frequently observed in the study and we consider this to be a principal contributory factor to the resultant musculoskeletal problems reported by the dental community in this and previous studies.

Regarding Dr Paul's comment about the positioning of the back rest: we consider that the back rest may not be an important factor influencing posture in this study. In the observation of 21 dentists across the West Midlands and around 200 students in the School of Dentistry - University of Birmingham, all using a CS, none of them were actively using their back rests while treating patients. The BS used in this study does not have a back rest; however, the design is such that it promotes good posture.

In response to the comment about sitting with thighs widely splayed on the BS: the BS allows the hips to fall into a supported abducted position and the angle adopted by each user will vary slightly, but will be within the 'normal range of movement'. This position is encouraged for increasing the blood supply to the head of the femur and for maintaining the length of the piriformis muscle. Sitting with slight abduction and external rotation of the hips is a natural position and seen as the position adopted by many when sitting.

Finally in response to the comment about seat height: we agree that seat height is an important factor influencing the working posture. Dr Paul has suggested that the CS has to be high enough for a dentist to put the mouth in his/her focal distance. The BS, like conventional 
seats, may readily be adjusted to the correct height, but the dental chair also needs to be adjusted relative to the dental seat height in order to obtain optimal vision of the tooth being operated on. This lack of sufficient raise may occur in a minority of older chairs but was not seen in this study.

We again thank Dr Paul for his comments regarding this article.

DOI: $10.1038 / b d j .2008 .110$

\section{SWEET NOTHINGS}

Sir, I must take issue with R. Cottrell's letter on sugar and health (BDJ 2007; 203: 560-561). He is incorrect to refer to 'the review of evidence of effectiveness of oral health promotion methods by Professor Liz Kay for the Health Development Agency', which he then references to an article in the $B D J$. The report, published in $1997,{ }^{1}$ was in fact commissioned by the Health Education Authority and was co-authored by Dr Elizabeth Kay and Professor David Locker. Both Dr Richard Watt and I wrote commentaries. The exact statement that the book contains on sugar (in the executive summary) runs as follows:

'Attempts to control individuals' consumption of sweet foods and drinks are generally not satisfactorily evaluated. However, when such interventions are directed towards individuals, they appear to be of limited value.'

The authors conclude that the most cost-effective means of delivering fluoride to the population should be the method of choice for promoting oral health through caries reduction.

However, looking at the broader issue of food and health today, the advice of the Food Standards Agency (FSA) is to cut down on sugar: ${ }^{2}$

'Most people in the UK are eating too much sugar. We should all be trying to eat fewer foods containing added sugar, such as sweets, cakes and biscuits, and drinking fewer sugary soft and fizzy drinks.

'Having sugary foods and drinks too often can cause tooth decay, especially if you have them between meals. Many foods that contain added sugar can also be high in calories so cutting down could help you control your weight.'

Irrespective of whether this advice is endorsed by the Sugar Bureau, it is part of the FSA's ' 8 tips for eating well', and so should be kept in mind by health professionals. If dental care professionals remain in any doubt about what to say to their patients in the surgery, Delivering better oral health ${ }^{3}$ recommends that everyone is advised to reduce the frequency and amount of sugary foods and drinks consumed.

C. Stillman-Lowe

Independent Oral Health Promotion Adviser

1. Kay E J, Locker D. Effectiveness of oral health promotion: a review. London: Health Education Authority, 1997.

2. http://www.eatwell.gov.uk/healthydiet/ eighttipssection/8tips/

3. Department of Health and the British Association for the Study of Community Dentistry. Delivering better oral health: an evidence-based toolkit for prevention. London: Department of Health, 2007.

DOI: 10.1038/bdj.2008.111

\section{TMJ DISLOCATION}

Sir, we would like to report a case of temporomandibular joint (TMJ) dislocation which occurred during administration of an inferior alveolar nerve block.

A 28-year-old male patient was being administered inferior alveolar nerve block by his dentist for a class II filling in the 36 , when the patient felt a click in the right TMJ, with associated pain and inability to close his mouth. A diagnosis of unilateral TMJ dislocation was made, however, no attempt was made to reduce the dislocation and the patient was referred to the Accident and Emergency department.

The dislocation was spontaneous. The patient did not have any previous history of clicking, subluxation, trauma or dislocation of the TMJ.

An orthopantogram was performed (Fig. 1) and the patient was referred on to the maxillofacial team. Clinical history and examination was not suggestive of connective tissue disorders like Ehler danlos or Marfan's syndrome.

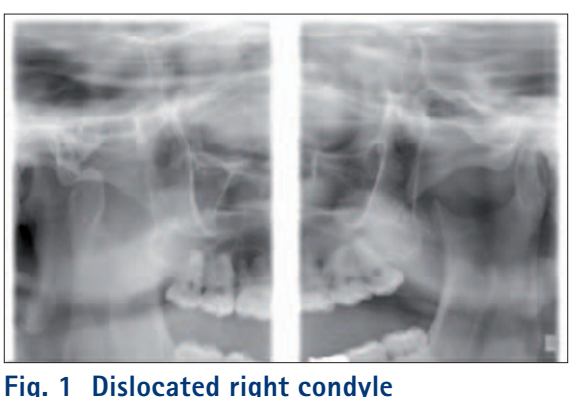

Fig. 1 Dislocated right condyle
The patient was in considerable pain. There was a palpable deformity and the TMJ was low on the same side.

Two millilitres of local anaesthetic was infiltrated around the right TMJ and the dislocation was reduced with considerable effort using a combined intraoral and extra oral technique. No sedation was required. The patient was advised not to open his mouth wide and to support his jaw. He was subsequently discharged with an analgesic prescription and warned about further risk of dislocations.

Non-traumatic dislocations of the TMJ occur most commonly while yawning, eating, dental treatment, endoscopy ${ }^{1}$ and oral intubation. ${ }^{2}$ Most dental treatments require the patient to open his or her mouth wide for a long period of time, which makes it likely for the dislocation to occur in the dental surgery. The risk of dislocation can be minimised by asking the patient to bite on a mouth prop and ensuring adequate rest during prolonged treatment.

In this particular patient, four hours had elapsed since the dislocation during which time the patient had been in a lot of discomfort. Spasm of the masseter and pterygoid muscles worsens as time progresses, rendering reduction difficult. In addition, complications of reduction such as fracture of the condyle and the articular eminence are rare if reduction is achieved early using the correct techniques.

All the tools that are required to reduce a non-traumatic dislocation which occurs in the dental surgery are within easy reach of a general dental practitioners ie local anaesthetic, panorex and if necessary sedation. It is far easier to relocate it immediately and the morbidity is much reduced if the dislocation is reduced as soon as possible. If needed, the patient may then be referred on to a maxillofacial unit.

Reduction of dislocated TMJ is being taught as a mandatory skill for physicians working in the emergency department. During dental school, the theoretical management of TMJ dislocation is covered in the curriculum. However, no attempt is made to train using mannequins or simulators to teach practical skills at reduction. 
We would like to emphasise the need to include teaching practical techniques in undergraduate dental education.

M. A. Nusrath, J. R. Adams, D. R. Farr, D. G. Bryant Middlesbrough

1. Mangi Q, Ridgway P F, Ibrahim Z, Evoy D. Dislocation of the mandible. Surg Endosc 2004; 18: 554-556.

2. Ting J. Temporomandibular joint dislocation after use of a laryngeal mask airway. Anaesthesia 2006; 61: 201.

DOI: 10.1038/bdj.2008.112

\section{RIGA-FEDE DISEASE}

Sir, we would like to report an unusual case of sublingual and ventral tongue ulceration, which was present for two weeks, in a six-week-old female Caucasian baby. The mother was concerned about her baby's intraoral discomfort and difficulty with feeding. Examination revealed a distressed baby with a lower right mandibular tooth of whitish colour and shape resembling a primary incisor, with grade II mobility. The tongue was slightly raised at rest with an $8 \times 10 \mathrm{~mm}$ indurated and tender ulcer on the ventral surface/floor of the mouth extending from the tip to the lingual fraenum (Fig. 1). The medical history was clear. As the tooth was the suspected cause of the ulceration and pain it was extracted and this was completed using topical anaesthetic. On review ten days later, examination revealed complete healing of the ulcer and the extraction socket. The baby appeared to be much more content and the mother reported that her baby was feeding normally.

Ulceration of the ventral surface of the tongue in newborn babies or infants is most commonly related to natal or neonatal teeth, as illustrated in this case. ${ }^{1,2}$ We believe this particular type of ulceration to be a case of Riga-Fede disease ${ }^{3}$ this was initially described by Riga in 1881 and Fede in $1890^{4}$ and has been reported since, but not widely in the British literature. Typically, the benign lesion begins as an ulcerated area on the ventral surface of the tongue exposed to repeated trauma from a natal tooth. ${ }^{2}$ It may progress to an enlarged fibrous mass and has appearances that have been described as those of a traumatic

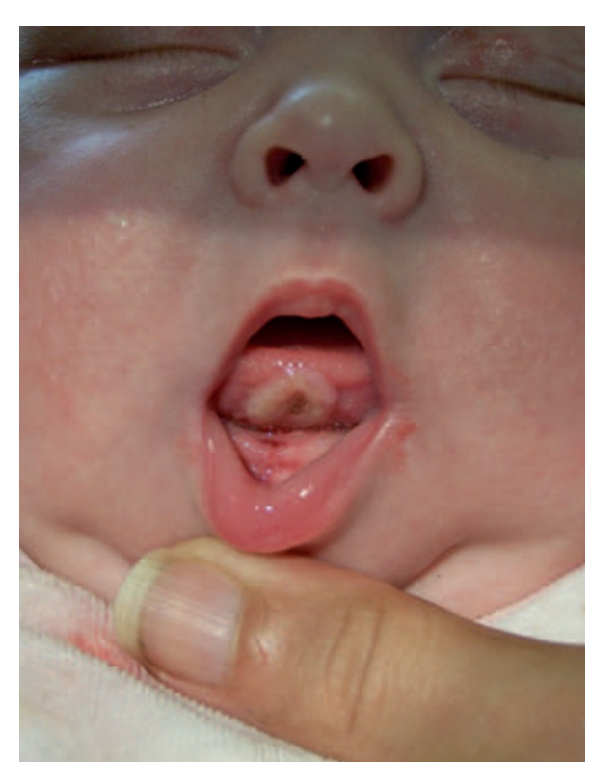

Fig. 1 Ulcer on the ventral surface/floor of the mouth

ulcerative granuloma (with stromal eosinophilia) ${ }^{5}$ (Fig. 1). Such ulceration can also be seen in infants with primary teeth and a tongue thrusting habit and in children with familial dysautonomia (insensitivity to pain). ${ }^{6}$ Recognition of these clinical signs may also be an indicator of neurological disorders.

Treatment of Riga-Fede disease has varied from excision of the lesion, to smoothening of the sharp incisal edges or rounding of the sharp edges of the tooth with composite increments, the latter being where mild to moderate ulceration occurs. ${ }^{4}$ Where the ulceration is large and interfering with feeding, removal of the tooth can be beneficial, as in this case.

D. Jariwala, R. M. Graham, T. Lewis

By email

1. Goho C. Neonatal sublingual traumatic ulceration (Riga-Fede disease): reports of cases. J Dent Child 1996; 63: 362-364.

2. Hedge R J. Sublingual traumatic ulceration due to neonatal teeth. J Indian Soc Pedod Prev Dent 2005; 23: 51-52.

3. Baghdadi Z D. Riga-Fede disease: report of a case and review. J Clin Pediatr Dent 2001. 25: 209-213.

4. Slayton R. Treatment alternatives for sublingual traumatic ulceration (Riga-Fede disease). Pediatr Dent 2000; 22: 413-414.

5. Elzay R P. Traumatic ulcerative granuloma with stromal eosinophilia (Riga-Fede disease and traumatic eosinophilic granuloma). Oral Surg Oral Med Oral Pathol 1983; 55: 497-506.

6. Rakocz M, Frand M, Brand N. Familial dysatonomia with Riga-Fede's disease: report of case. ASDC J Dent Child 1987; 54: 57-59.

DOI: $10.1038 /$ bdj.2008.113 